\title{
Fast Track Extubation Post Coronary Artery Bypass Graft: A Retrospective Review of Predictors of Clinical Outcomes"
}

\author{
Shelly Bansal ${ }^{\text {\#\# } \text {, H. M. Thai }}{ }^{2}$, C. H. Hsu ${ }^{3}$, C. B. Sai-Sudhakar ${ }^{4}$, S. Goldman ${ }^{2}$, B. E. Rhenman ${ }^{5}$ \\ ${ }^{1}$ Department of Surgery, University of Arizona, Tucson, USA \\ ${ }^{2}$ Cardiology Section (1-111C), Southern Arizona VA Health Care System, Tucson, USA \\ ${ }^{3}$ Division of Epidemiology and Biostatistics, Mel \& Enid Zuckerman College of Public Health, \\ University of Arizona, Tucson, USA \\ ${ }^{4}$ Department of Cardiac Surgery, The Ohio State University, Columbus, USA \\ ${ }^{5}$ Department of Cardiothoracic Surgery, University of Arizona Health Sciences Center, Tucson, USA \\ Email: "shelly.bansal@osumc.edu
}

Received April 1, 2013; revised May 1, 2013; accepted May 8, 2013

Copyright (C) 2013 Shelly Bansal et al. This is an open access article distributed under the Creative Commons Attribution License, which permits unrestricted use, distribution, and reproduction in any medium, provided the original work is properly cited.

\begin{abstract}
Introduction: Fast track (FT) cardiac surgery and early extubation (EE) are aimed at safe and effective rapid post-operative progression to discharge, and have been practiced for more than two decades. Their goal is to optimize patient care perioperatively in order to decrease costs without negatively affecting morbidity and mortality. However, the factors that predict successful EE are poorly understood, and patients with significant co-morbidities are frequently excluded from protocols. We hypothesize that independent of disease severity, early extubation leads to shorter hospital stays and can be performed safely without negatively affecting outcomes. Materials and Methods: We performed a retrospective review of 919 patients who underwent coronary artery bypass grafting (CABG) at the Southern Arizona Veteran's Affairs Health Care System medical center over 7 years. We collected pre-operative data regarding patients' NYHA classification, presence and severity of cerebral vascular disease, peripheral vascular disease, pulmonary disease, diabetes and hypertension. Intra-operative variables were also recorded including ASA scores, ischemic times, and time to extubation. Finally, post-operative variables such as rates of reintubation and tracheotomy, and both length of ICU and total hospital stay were also compared. Results: Prolonged periods of ischemia were found to predict a delayed extubation $(\mathrm{HR}=0.992 ; \mathrm{CI}=0.988-0.997, \mathrm{p}=0.0015)$ while small body surface area $(\mathrm{HR}=1.57 ; \mathrm{CI}=1.13,2.17, \mathrm{p}=$ $0.007)$ and higher pre-operative functional status of the patient, such as independent versus dependent status $(\mathrm{HR}=1.68$; $\mathrm{CI}=1.30-2.16, \mathrm{p}<0.0001)$, or partially dependent to fully dependent status $(\mathrm{HR}=1.33$; $\mathrm{CI}=1.03-1.70, \mathrm{p}=0.03)$ were found to be associated with earlier extubation. The early extubation (EE) group (those extubated in less than the median 7.3 hours) had an average hospital stay of $5.1 \pm 4.0$ days, versus $7.8 \pm 8.1$ days in the delayed group ( $>4$ hours), $\mathrm{p}<0.0001$. The EE group also experienced shorter ICU stays by about 1 day (EE, $1.9 \pm 4.2$ v. conventional, $3.8 \pm 17.3$, $\mathrm{p}=0.02)$. There were no differences in rates of tracheotomy or re-intubation between groups. Conclusions: In our study population, pre-operative functional class and total body surface area predicted those patients able to tolerate early extubation after cardiac surgery. Prolonged ischemia resulted in delayed extubation. Patients that were extubated in less than 4 hours had shorter ICU and hospitalization stays, while there was no significant difference between the two groups in rate of reintubation or tracheotomy.
\end{abstract}

Keywords: Coronary Artery Bypass; Fast Track; Early Extubation; Postoperative Period; Intensive Care; Intubation; Treatment Outcome; Airway Extubation

\footnotetext{
*This study was performed at the Southern Arizona VA Health Care System and was supported by grants from the Department of Veterans Affairs, the WARMER Foundation, The Hansjörg Wyss Foundation, the Arizona Biomedical Research Commission, and the Biomedical Research and Education Foundation of Southern Arizona.

"Corresponding author.
}

\section{Introduction}

Today, the need for high quality and cost-effective care of surgical patients has lead to an increased focus on methods for decreasing length of ICU and total hospital 
stays. In regards to cardiac surgery, approximately 500,000 coronary artery bypass graft (CABG) procedures are performed annually in the United States at a substantial cost to the healthcare system [1]. As a result the development of fast track (FT) and early extubation (EE) management protocols have been developed to provide safe, effective and rapid post-operative progression to discharge of post CABG patients [1]. This approach has been practiced for more than two decades beginning back in the 1990's ,when “fast-track” protocols were developed and since associated with decreased lengths of stay (LOS) without negatively affecting outcomes [2,3] and potentially lowering rates of ventilator associated pneumonia [4].

Despite these benefits, the key factors associated with successful EE and FT post-operative management are poorly understood. Consequently, it is unclear if patients with significant comorbidities should be excluded from EE. The purpose of this paper is to review and evaluate the safety and efficacy of early extubation in patients undergoing coronary artery bypass grafting in terms of their preoperative disease severity.

\section{Materials and Methods}

Institutional Review Board approval was obtained prior to the study. We performed a retrospective chart review of all patients who underwent an isolated coronary artery bypass grafting (CABG) at the Southern Arizona Veteran's Affairs Health Care System medical center over 7 years. All patients undergoing additional valve replacement at the time of surgery were excluded from analysis. We collected demographic data including age, gender, height, weight, and body surface area. The patient's preoperative condition and disease severity was documented based on the following variables: cerebral vascular disease, NYHA classification, presence of pulmonary disease, diabetes, hypertension and degree of peripheral vascular disease.

Data collected regarding the intra-operative course included: ASA scores, ischemic times, number of grafts implanted and time to extubation. Extubation times were noted and calculated from the time patient left the operating room to time of endotracheal tube (ET) removal. Additional data regarding re-intubation rates, requirement of tracheotomy, and length of ICU and total hospital stay were also recorded.

Additionally, a Charlson co-morbidity index, a previously validated scoring system aimed at predicting the ten-year mortality of patients with chronic illnesses and co-morbid conditions including heart disease [5,6] was calculated for each patient.

Patient demographics were calculated using means and standard errors. Statistically significant changes in length of intubation over time were detected via a one-way ANOVA. A Cox proportional hazards model which is a statistical technique for exploring the relationship between the survival of a patient and several explanatory variables was used to identify variables that predicted longer intubation times [7]. Finally, comparison between early extubation groups and delayed groups was performed using Fisher's exact test for discrete variables and by two tailed t-test with unequal variances for continuous variables. The significance threshold for all tests was defined as $<0.05$.

\section{Results}

A total of 919 patient records met the study inclusion criteria. Table 1 shows patient demographics. The mean patient age was $64.9 \pm 8.6$ years. Over $98 \%$ were male and had a mean weight of $198.6 \pm 38.8$ lbs. Comorbidities were common in the cohort; $96.6 \%$ of the patients were ASA class 4 , and $37.7 \%$ had been diagnosed with chronic obstructive pulmonary disease and 34.6\% with diabetes. Additionally $21.6 \%$ had a history of smoking and $47.5 \%$ had a history of prior myocardial infarction.

Table 1. Demographics of study cohort $(N=919)$. The mean age was $64.9 \pm 8.6$, majority of patients were males. The mean weight was $198.6 \pm 38.8 \mathrm{lbs}$. $21.6 \%$ were current smokers; there were $37.7 \%$ with a diagnosis of chronic obstructive pulmonary disease (COPD) prior to surgery. $47.5 \%$ had a prior myocardial infarction (MI). 34.6\% had diabetes.

\begin{tabular}{cc}
\hline Category & Mean \pm SD (\%) \\
\hline Age & $64.9 \pm 8.6$ \\
Male & $98.2 \%$ \\
Weight (lbs.) & $198.6 \pm 38.8$ \\
Current Smoker & $198(21.5)$ \\
COPD & $346(37.7)$ \\
Diabetes & \\
No & $601(65.4)$ \\
Type I & $159(17.3)$ \\
Type II & $159(17.3)$ \\
Prior MI & \\
No & $482(52.5)$ \\
Yes & $437(47.5)$ \\
Stroke & $8(0.9)$ \\
ASA & \\
1 - 2 & $2(0.2)$ \\
3 & $29(3.2)$ \\
4 & $888(96.6)$ \\
PVD & $205(22.3)$ \\
Any Complication & $90(9.8)$ \\
Priority & \\
1 & $692(75.3)$ \\
2 & $167(18.2)$ \\
3 & $60(6.5)$ \\
\hline
\end{tabular}


To better understand the severity of patient comorbidity and their long-term mortality risk, we calculated the mean Charlson score for the patients included in the study by year of surgery. The results of these calculations are given in Table 2. Overall, the mean Charlson score was $5.99 \pm 2.54$. Additionally, over time there was a statistically significant trend toward decreasing Charlson score as determined by one-way ANOVA ( $<<0.0001)$.

A log-rank test was performed for time to extubation by year of surgery. The p-value for both overall and trend log-rank tests for surgical years was $<0.0001$, indicating that there were significant differences in time to extubation between surgical years. As shown in Figure 1, it is possible to see a clear decrease in extubation time over the study period. This trend coincides becomes most apparent around 2004, where we see a distinct transition from a linear decrease in Extubation-free probability over time to a high probability of extubation within 7 hours of surgery. This coincides with a decisive change in management at our institution, to move to more rapid extubation in our patients following coronary artery bypass graft procedures.

The median time to extubation for all records was less than 7.3. We then divided the records into two groups, the EE group (extubated in less than 7.3 hours) and the

Table 2. Mean Charlson score by surgical year. The overall score calculated for this population was $5.99 \pm 2.54$. The overall trend appears to be a decreasing Charlson score over time. Based on one-way ANOVA, the p-value for surgical year is $<0.0001$ indicating that the mean Charlson score significantly varies by surgical year. According to Turkey's HSD post hoc test, $1995>(1998,1999,2000,2005$, 2006, 2007); 1996 and $1997>(1998,1999,2000,2001,2002$, 2004, 2005, 2006, 2007).

\begin{tabular}{ccccc}
\hline Year & N & Mean & SD & Range \\
\hline 1995 & 36 & 7.22 & 2.21 & $4-12$ \\
1996 & 180 & 7.08 & 2.09 & $3-13$ \\
1997 & 157 & 7.03 & 2.54 & $1-14$ \\
1998 & 130 & 5.58 & 2.77 & $0-14$ \\
1999 & 115 & 5.30 & 2.64 & $0-12$ \\
2000 & 116 & 5.51 & 2.38 & $1-11$ \\
2001 & 178 & 5.87 & 2.62 & $1-12$ \\
2002 & 152 & 5.80 & 2.31 & $0-12$ \\
2003 & 135 & 6.16 & 2.54 & $1-13$ \\
2004 & 125 & 5.94 & 2.55 & $1-15$ \\
2005 & 135 & 5.19 & 2.30 & $1-11$ \\
2006 & 142 & 5.66 & 2.50 & $1-14$ \\
2007 & 37 & 5.27 & 2.63 & $1-13$ \\
\hline
\end{tabular}

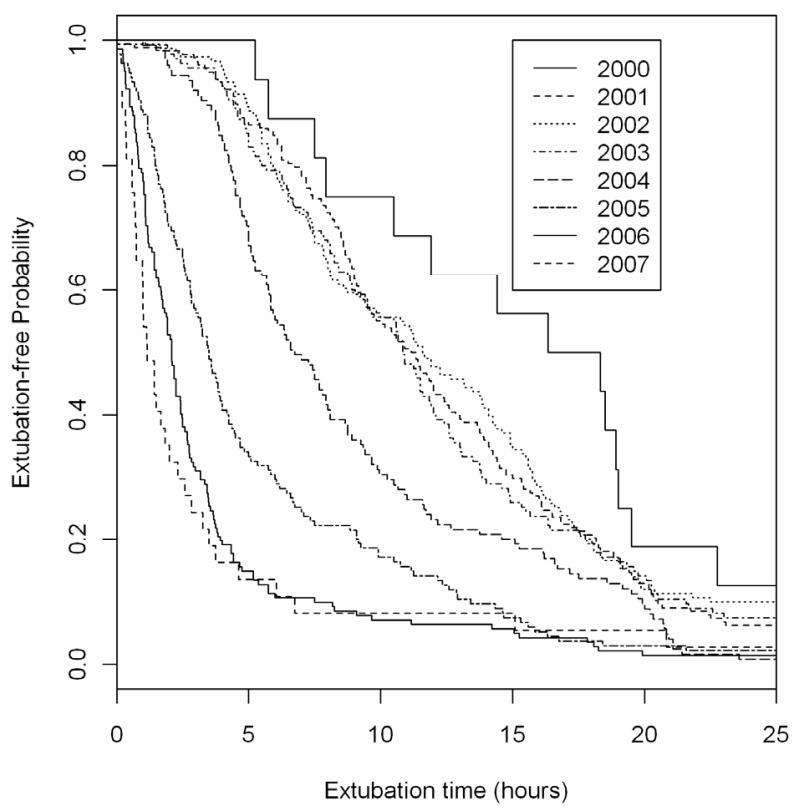

Figure 1. Kaplan-Meier curves for time to extubation by surgical year showing the extubation free probability on the $Y$ axis and time to extubation on the $X$ axis. Each curve represents a single surgical year, with a trend toward shorter extubation times over time. This was a statistically significant difference in time to extubation by surgical year as indicated with a $\log$ rank p-value of $<\mathbf{0 . 0 0 0 1}$.

conventional group (extubated in more than 7.3 hours). Comparing the two groups we found statistically significant differences in length of ICU and total hospital stay. The early extubation group had an average hospital stay of $5.10 \pm 4.03$ days, versus $7.78 \pm 8.14$ days in the delayed group (two tailed t-test, $\mathrm{p}<0.0001$ ). The early extubation group also had shorter ICU stays than the delayed extubation group by about 1 day (1.92 \pm 4.17 vs. $3.80 \pm 17.29, p=0.02)$. Notably, there was no significant difference in the tracheotomy rates between the two groups nor was there any difference in the frequency of re-intubation.

Table 3 shows the results of the Cox proportional hazard model adjusted for age and gender to determine the variables that were predictive of time to extubation. These results show that longer ischemic times predicted delayed extubation $(\mathrm{HR}=0.992$; $\mathrm{CI}=0.988-0.997, \mathrm{p}=0.0015)$. Additionally, lower body surface area $(\mathrm{HR}=1.57$; $\mathrm{CI}=$ 1.13, 2.17, $\mathrm{p}=0.007$ ) and more independent patient functional status prior to surgery, classified as independent versus fully dependent $(\mathrm{HR}=1.68$; $\mathrm{CI}=1.30-2.16$, $\mathrm{p}<0.0001)$, or partially dependent versus fully dependent $(\mathrm{HR}=1.33$; $\mathrm{CI}=1.03-1.70, \mathrm{p}=0.03)$ were associated with earlier extubation times.

\section{Discussion}

Our study's main purpose was to review the safety and 
Table 3. Stratified (surgical year as strata) Cox proportional hazards model for risk of extubation adjusting for age and gender. Ischemic times $(\mathrm{HR}=\mathbf{0 . 9 9 2} ; \mathrm{CI}=\mathbf{0 . 9 8 8}-\mathbf{0 . 9 9 7}, \mathrm{p}=\mathbf{0 . 0 0 1 5})$ were found to predict a delayed extubation. While body surface area $(H R=1.57 ; C I=1.13,2.17, p=0.007)$ and the functional status of the patient, classified as independent versus fully dependent status $(\mathrm{HR}=1.68 ; \mathrm{CI}=1.30-2.16, \mathrm{p}<0.0001)$, or partially dependent to dependent status $(\mathrm{HR}=1.33 ; \mathrm{CI}=1.03$ $1.70, p=0.03$ ), were independent variables associated with earlier extubation times. Ischemic Time, ISCT; Functional Status, FST, 1 = independent, 2 = partially independent, 3 = fully dependent; Body Surface Area, BSA.

\begin{tabular}{|c|c|c|c|}
\hline Variable & Hazard ratio & $95 \% \mathrm{CI}$ & p-value \\
\hline CABG vessels & & $\mathrm{p}$-value $=0.0861$ & \\
\hline 1 vs. 5 & 1.63 & $(0.87,3.06)$ & 0.1290 \\
\hline 2 vs. 5 & 1.79 & $(1.00,3.19)$ & 0.0484 \\
\hline 3 vs. 5 & 1.59 & $(0.90,2.82)$ & 0.1122 \\
\hline 4 vs. 5 & 1.36 & $(0.75,2.48)$ & 0.3107 \\
\hline ISCT & 0.992 & $(0.988,0.997)$ & 0.0015 \\
\hline ASA & & $\mathrm{p}$-value $=0.5036$ & \\
\hline $1 \mathrm{vs} .4$ & 1.60 & $(0.39,6.49)$ & 0.5125 \\
\hline 3 vs. 4 & 1.21 & $(0.83,1.76)$ & 0.3249 \\
\hline COPD & 0.91 & $(0.79,1.04)$ & 0.1565 \\
\hline Current Smoker & 0.92 & $(0.78,1.09)$ & 0.3600 \\
\hline FST & & p-value $<0.0001$ & \\
\hline 1 vs. 3 & 1.68 & $(1.30,2.16)$ & $<0.0001$ \\
\hline 2 vs. 3 & 1.33 & $(1.03,1.70)$ & 0.0276 \\
\hline BSA & 1.57 & $(1.13,2.17)$ & 0.0067 \\
\hline Ethnicity (Latino vs. others) & 0.65 & $(0.32,1.29)$ & 0.2169 \\
\hline Race (white vs. others) & 0.71 & $(0.40,1.29)$ & 0.2631 \\
\hline
\end{tabular}

Table 4. Frequency of clinical outcome in groups with extubation less than the median of 7.3 hours compared to a group of patients with extubation times greater than 7.3 hours.

\begin{tabular}{|c|c|c|c|}
\hline & Extubation $\leq 7.33^{+}(N=456)$ & Extubation $>7.33(463)$ & p-value ${ }^{*}$ \\
\hline Tracheotomy & $1 / 456(0.2 \%)$ & $0 / 460(0.0 \%)$ & 0.50 \\
\hline Reintubation & $3 / 279(1.1 \%)$ & $0 / 57(0.0 \%)$ & 1.000 \\
\hline Total Stay in days $($ mean $\pm S D)$ & $5.10 \pm 4.03$ & $7.78 \pm 8.14$ & $<0.0001$ \\
\hline ICU Stay in days (mean $\pm S D)$ & $1.92 \pm 4.17$ & $3.80 \pm 17.29$ & $<0.05$ \\
\hline
\end{tabular}

* p-value for categorical variables derived from a Fisher's exact test; for continuous variables derived from a two tailed t-test with unequal variances.

efficacy of early extubation in the post-operative management of CABG patients in the Southern Arizona Veteran's Affairs Health Care System over a seven-year period. We compared ICU and total hospital length of stay, as well as complication rates in extubation early patients $(<7.3 \mathrm{~h})$ or in those extubated later $(>7.3 \mathrm{~h})$ (Table 4). The main principle underlying the fast track protocols pioneered in the 1990's is that rapid post-operative progression can reduce length of stay and cost [8-10], without negatively affecting morbidity and mortality [11-14].

Further, the protocol offers potential benefits to pa- tients including a quick return to normal activity, decreased rates of ventilator associated pneumonia [4], other hospital associated infection such as surgical site infection, urinary tract, and central line-associated bloodstream infections $[15,16]$, and complications of immobility like deep venous thrombosis and pulmonary embolism.

We found that early extubation was associated with a shorter ICU and total hospital stay, without differences in rates of tracheotomy or reintubation. These findings suggest that in our patient demographic, fast track post-op- 
erative management is both safe and effective. Similar findings have been previously reported in the literature [11-14], including a recent Cochrane Review, were a meta-analysis of six clinical trials including 871 total cases found no differences in intensive care mortality, 30-day mortality, or rates of reintubation in individuals extubated less than 8hours compared to those undergoing conventional management [17]. In confirming the safety and efficacy of fast track post operative management with early extubation, our data show that at our institution a concerted change in management can reproducibly yield high quality low cost outcomes and confirm the positive improvement in patient care.

While reintubation rates were not clearly affected by extubation time, patients who remained on the ventilator longer than the median extubation time were often ventilated for extended periods, defined here as greater than 48 hours. These individuals experienced a particularly complicated post-operative course, and may represent a subpopulation that is not appropriate for early extubation. Further factors identified as predictive of longer extubation times included longer ischemic times, higher body surface area and more dependent functional status. The association with body surface area is likely related to the reported risk of failure of early extubation in obese patients $[18,19]$. This suggests that these factors may be important in determining patient appropriateness for early extubation.

Of note, in our study neither the number of involved coronary arteries, the incidence of general preoperative comorbidity, nor respiratory pathology specifically chronic obstructive pulmonary disease (COPD), had a demonstrable effect on time to extubation or length of stay. This indicates that in these patients neither multi-vessel coronary artery disease, nor a history of respiratory or other systemic illness is a contraindication to early extubation and fast track post-operative management. However, alternative studies have reported a significant association between number of diseased arteries, ejection fraction and EuroSCORE, a severity index for cardiovascular disease, and successful early extubation following CABG procedure [20]. Together, these suggest that no specific illness or co-morbidity is a complete contraindication for fast-track post-operative management, but rather that an aggregate assessment of functional status is more meaningfully predictive of time to extubation.

Additionally, our study identified several notable time trends. First, there was a significant decrease in the mean Charlson index over time. There was also a trend toward decreasing times to extubation based on surgical year. These findings likely represent changes in management of coronary artery disease, as patients are undergoing CABG procedures earlier, with less comorbidity [21]. Similarly, the decrease in extubation times likely reflects the general acceptance of early extubation management. At our institution, this has resulted from a multi-factorial effort to shorten our extubation times through enhancements in preoperative teaching, anesthetic care, nursing coordination and surgeon involvement. We believe that this approach is preferable, as early extubation can lead to early mobilization, decreased risk of nosocomial infection, sternal wound-healing complication and other morbidity.

There are notable limitations to the study. First, 98\% of the patients were male. While this accurately reflects the demographics of the treatment population at a VA hospital, the results may not apply to female patients. In comparison we refer to Guller et al. performing a large study of early extubation consisting of 6446 patients in which $35.3 \%$ were female. The researchers found the overall mean post-CABG intubation time was 9.8 (SD 5.7) hours (median, 8.5 hours; 25th percentile, 5.6 hours; 75th percentile, 13.5 hours). Twenty-nine percent of patients overall were extubated within 6 hours after CABG surgery; $40.6 \%$ were extubated within 6 to 12 hours and 30.4\% were extubated between 12 and 24 hours. Shorter post-CABG extubation times were significantly associated with better patient outcomes.

The authors concluded similarly to our study that early extubation is associated with significantly shorter postoperative length of stay without increased mortality or frequency of reintubation. The authors also found early extubation to be safe in the overall patient population as well as in the subsets of patients below and above 75 years of age, in male versus female patients, and in patients with and without major comorbidities [22].

Additionally, as a retrospective review it most directly identifies patient variables currently associated with clinical decisions about post-operative management. It does not provide direct comparative information about morbidity and mortality in patients prospectively assigned to either early extubation or conventional management. However, a randomized trial with 100 eligible patients by Silbert et al. showed that early extubation did not lead to higher rates of re-intubation [17]. Similarly, Cheng et al. prospectively followed 120 patients undergoing coronary artery bypass grafting, and also found no correlation between early extubation and reintubation rates [11]. Together with our data, this suggests that for the vast majority of patients fast-track management with early extubation may be associated with shorter ICU and total hospital stays and reduced costs, regardless of Charlson index or other comorbidity.

\section{Conclusion}

In summary, our data was consistent with the current literature indicating that fast track cardiac surgery is a 
safe and viable protocol that improves both patient care and resource utilization. Additionally, we were able to identify independent predictors of early extubation, including decreased ischemic times, lower body surface area and more independent functional status. This information may be used to guide patient management and allow us to develop a treatment algorithm that will standardize care and allow early extubation to be standard management for the majority patients, regardless of other significant comorbidities.

\section{Acknowledgements}

This work was supported by grants from the Department of Veterans Affairs, the WARMER Foundation, The Hansjörg Wyss Foundation, the Arizona Biomedical Research Commission, and the Biomedical Research and Education Foundation of Southern Arizona.

\section{REFERENCES}

[1] Swaminathan, Madhav, P. Bute, G. Barbara, et al., "Increasing Healthcare Resource Utilization after Coronary Artery Bypass Graft Surgery in the United States,” Circulation: Cardiovascular Quality and Outcomes, Vol. 2, No. 4, 2009, pp. 305-312. doi:10.1161/CIRCOUTCOMES.108.831016

[2] C. Hawkes, D. R. Foxcroft and P. Yerrell, "Clinical Guideline for Nurse-Led Early Extubation after Coronary Artery Bypass: An Evaluation,” Journal of Advanced Nursing, Vol. 66, No. 9, 2010, pp. 2038-2049.

[3] R. U. Pande, N. D. Nader, H. W. Donias, et al., "Review: Fast-Tracking Cardiac Surgery," Heart Surgery Forum, Vol. 6, No. 4, 2003, pp. 244-248.

[4] M. J. London, A. L. Shroyer, V. Jernigan, et al., "FastTrack Cardiac Surgery in a Department of Veterans Affairs Patient Population,” The Annals of Thoracic Surgery, Vol. 64, No. 4, 1997, pp. 134-141. doi:10.1016/S0003-4975(97)00248-8

[5] M. E. Charlson, P. Pompei, K. L. Ales, et al., “A New Method of Classifying Prognostic Comorbidity in Longitudinal Studies: Development and Validation,” Journal of Chronic Diseases, Vol. 40, No. 5, 1987, pp. 373-383. doi:10.1016/0021-9681(87)90171-8

[6] J. McDonald, "Handbook of Biological Statistics," 2nd Edition, Sparky House Publishing, Baltimore, 2009.

[7] J. Walters Stephen, "Reader in Medical Statistics, What Is a Cox Model?” Hayward Medical Communications, 2009. www.whatisseries.co.uk

[8] D. C. Cheng, "Impact of Early Tracheal Extubation on Hospital Discharge," Journal of Cardiothoracic and Vascular Anesthesia, Vol. 12, 1998, pp. 35-44.

[9] A. L. Corsetti and D. Perry, "A Comprehensive Approach to Facilitating the Recovery of Cardiac Surgery Patients," Journal of Cardiovascular Nursing, Vol. 12, No. 3, 1998, pp. 82-90.

[10] D. R. Zevola and B. Maier, "Improving the Care of Car- diothoracic Surgery Patients through Advanced Nursing Skills,” Critical Care Nurse, Vol. 19, No. 1, 1999, pp. 34-44.

[11] J. Butler, G. L. Chong, R. Pillai, et al., "Early Extubation after Coronary Artery Bypass Surgery: Effects on Oxygen Flux and Haemodynamic Variables," The Journal of Cardiovascular Surgery (Torino), Vol. 30, 1992, pp. 276280.

[12] J. L. Chong, C. Grebenik, M. Sinclair, et al., "The Effect of a Cardiac Surgical Recovery Area on the Timing of Extubation," Journal of Cardiothoracic and Vascular Anesthesia, Vol. 7, No. 2, 1993, pp. 137-141. doi:10.1016/1053-0770(93)90205-Y

[13] D. C. Cheng, "Pro: Early Extubation after Cardiac Surgery Decreases Intensive Care Unit Stay and Cost,” Journal of Cardiothoracic and Vascular Anesthesia, Vol. 9, No. 4, 1995, pp. 460-464. doi:10.1016/S1053-0770(05)80105-3

[14] D. C. Cheng, J. Karski, C. Peniston, et al., "Morbidity Outcome in Early Versus Conventional Tracheal Extubation after Coronary Artery Bypass Grafting: A Prospective Randomized Controlled Trial,” The Journal of Thoracic and Cardiovascular Surgery, Vol. 112, No. 3, 1996, pp. 755-764. doi:10.1016/S0022-5223(96)70062-4

[15] J. Y. Fagon, J. Chastre, A. Vuagnat, et al., "Nosocomial Pneumonia and Mortality among Patients in Intensive Care Units," Journal of the American Medical Association, Vol. 275, No. 11, 1996, pp. 866-869. doi:10.1001/jama.1996.03530350048033

[16] J. Y. Fagon, J. L. Trouillet and J. Chastre, "Nosocomial Pneumonia in Intensive Care Units,” La Presse Médicale, Vol. 25, 1996, pp. 1441-1446.

[17] C. A. Hawkes, S. Dhileepan and D. Foxcroft, "Early Extubation for Adult Cardiac Surgical Patients,” Cochrane Database of Systematic Reviews, Vol. 4, 2003, Article ID: CD003587.

[18] J. L. Parlow, R. Ahn and B. Milne, "Obesity Is a Risk Factor for Failure of 'Fast Track' Extubation Following Coronary Artery Bypass Surgery,” Canadian Journal of Anesthesia, Vol. 53, No. 3, 2006, pp. 288-294. doi:10.1007/BF03022217

[19] A. El Solh, A. Aquilina, et al., "Noninvasive Ventilation for Prevention of Post-Extubation Respiratory Failure in Obese Patients,” The European Respiratory Journal, Vol. 28, No. 3, 2006, pp. 588-595. doi:10.1183/09031936.06.00150705

[20] M. Sato, E. Suenaga, S. Koga, et al., "Early Tracheal Extubation after On-Pump Coronary Artery Bypass Grafting," Annals of Thoracic and Cardiovascular Surgery, Vol. 15, No. 4, 2009, pp. 239-242.

[21] B. S. Silbert, J. D. Santamaria, J. L. O’Brien, et al., "Early Extubation Following Coronary Artery Bypass Surgery: A Prospective Randomized Controlled Trial. The Fast Track Cardiac Care Team,” Chest, Vol. 113, No. 6, 1998, pp. 1481-1488. doi:10.1378/chest.113.6.1481

[22] Guller, Ulrich, et al., "Outcomes of Early Extubation after Bypass Surgery in the Elderly," The Annals of Thoracic Surgery, Vol. 77, No. 3, 2004, pp. 781-788. 